\title{
AVIFAUNAL DIVERSITY IN THE SCRUB FOREST OF SRI LANKAMALLESWARA Wildlife SANCTUARY, ANDHRa PRADESh, INDIA
}

\author{
Sumant Mali ${ }^{1}$, Chelmala Srinivasulu ${ }^{2} \&$ Asad R. Rahmani ${ }^{3}$ \\ 1,3 Bombay Natural History Society, Hornbill House, Shaheed Bhagat Singh Road, Opp. Lion Gate, Mumbai, \\ Maharashtra 400001, India \\ ${ }^{2}$ Natural History Museum and Wildlife Biology \& Taxonomy Lab, Department of Zoology, University College of Science, \\ Osmania University, Hyderabad, Telangana 500007, India \\ ${ }^{2}$ Systematics, Ecology \& Conservation Laboratory, Zoo Outreach Organization (ZOO), No 12, Thiruvannamalai Nagar, \\ Saravanampatti-Kalapatti Road, Saravanampatti, Coimbatore, Tamil Nadu 641035, India \\ ${ }^{1}$ sumant_19_mali@yahoo.com, ${ }^{2}$ chelmalasrinivasulu@gmail.com (corresponding author), ${ }^{3}$ rahmani.asad@gmail.com
}

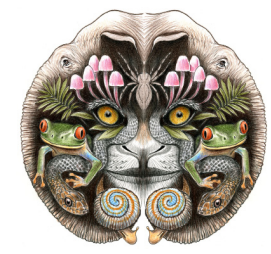

ISSN 0974-7907 (Online) ISSN 0974-7893 (Print)

OPEN ACCESS

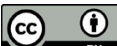

Abstract: A total of 115 bird species belonging to 47 families were recorded in the scrub forests of the Sri Lankamalleswara Wildlife Sanctuary, Andhra Pradesh, India in a survey carried out from May 2014 to April 2015. Of these, 107 species were resident and nine species were resident migrants. The scrub forests of peninsular India are equally important as other habitats for avifaunal assemblages.

Keywords: Birds, Eastern Ghats, Jerdon's Courser, protected area.

Birds are one of the best indicators of environmental quality of any ecosystem. Most of the birds have specific habitat requirements that change from season to season, and also show different species composition in different habitats. Scrub forest (whether it is a few isolated shrubs or young trees, or a dense thicket) is an important wildlife habitat. It is a natural part of other habitats, such as grassland and woodland, and an important component of the landscape. Diversity of avifauna is one of the most important ecological indicators to evaluate the quality of habitats. In general, owing to the destruction of natural habitats, anthropogenic disturbances, pollution and climate, the avifaunal diversity has been showing considerable variability both at spatial and temporal scales.

The conservation value of scrubland has been largely overlooked as major focus is invariably given to forests. This is despite the fact that scrub habitat is important for many species. Many birds feed in the open, but near enough to scrub, to be able to find refuge if danger threatens. In addition, scattered scrub in open sites is often very significant for nest sites and song-posts (Malcome 2007). In India, various studies have been carried out on scrubland bird species (Gandhi 1986, 2006; Johnsingh et al. 1987; Santharam 1989), however, only a few studies (Jeganathan 2005; Jeganathan et al.

DOI: http://doi.org/10.11609/jott.2720.9.9.10679-10691 | ZooBank: urn:Isid:zoobank.org:pub:9BA5C0BC-8B30-4891-963B-39CB16507055

Editor: P.O. Nameer, Kerala Agricultural University, Thrissur, India.

Date of publication: 26 September 2017 (online \& print)

Manuscript details: Ms \# 2720 | Received 13 April 2016 | Final received 08 August 2017 | Finally accepted 09 September 2017

Citation: Mali, S., C. Srinivasulu \& A.R. Rahmani (2017). Avifaunal diversity in the scrub forest of Sri Lankamalleswara Wildlife Sanctuary, Andhra Pradesh, India. Journal of Threatened Taxa 9(9): 10679-10691; http://doi.org/10.11609/jott.2720.9.9.10679-10691

Copyright: (c) Mali et al. 2017. Creative Commons Attribution 4.0 International License. JoTT allows unrestricted use of this article in any medium, reproduction and distribution by providing adequate credit to the authors and the source of publication.

Funding: This study was supported by Andhra Pradesh Forest Department, Government of Andhra Pradesh; Ministry of Environment, Forest and Climate Change, Government of India.

Competing interests: The authors declare no competing interests.

Acknowledgements: This work is part of a research project titled 'Conservation of Jerdon's Courser in Sri Lankamalleswara Wildlife Sanctuary' funded by Ministry of Environment Forest and Climate Change, Government of India and Andhra Pradesh Forest Department, Government of Andhra Pradesh. The authors wish to thank the Bombay Natural History Society, Mumbai for logistic support and Osmania University, Hyderabad for extending necessary facilities. The authors are also grateful to staff of the AP Forest Department and Rajendra, Rahim, Aslam, Srinu, Venkat Subaiah for their assistance during the field work.
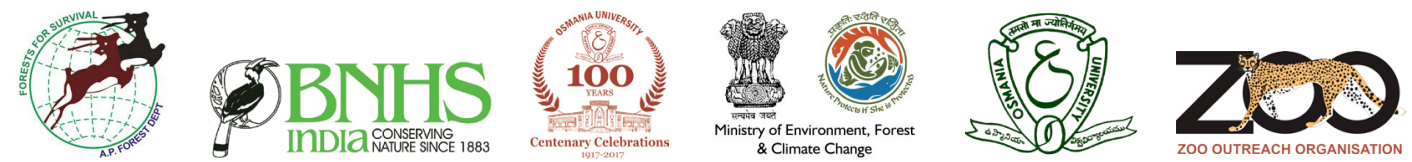
2008) have focused on the significance of scrub forest for scrubland bird species.

Sri Lankamalleswara Wildlife Sanctuary (SLWS), Kadapa (Cuddapah) District, Andhra Pradesh, India, falls under the Lankamallai hill ranges of the Eastern Ghats. Earlier studies on the avifauna of the scrub forests of SLWS were focused exclusively on the Critically Endangered Jerdon's Courser Rhinoptilos bitorquatus. There is a lack of information on the other scrubland birds of the sanctuary. This work aims to document the avifauna of scrub forest of SLWS.

\section{STUDY AREA}

SLWS lies between the Nallamalai and Sechachalam hill ranges in the central part of the Eastern Ghats. The sanctuary is located between $14^{\circ} 45^{\prime}$ to $15^{\circ} 12^{\prime} \mathrm{N}$ and $78^{\circ} 48^{\prime}$ to $79^{\circ} 6^{\prime} \mathrm{E}$ (Image 1 ). It is famous for the last known population for Critically Endangered Jerdon's Courser. The sanctuary ranges from about 137-784 m above mean sea level in elevation. The major types of forest in this sanctuary are southern tropical thorn and southern tropical dry deciduous (Champion \& Seth 1968). The sanctuary is bordered in the west by dense scrub and dry red sanders-bearing forest as well as southern dry mixed deciduous forests on the higher elevations of the Lankamallai hills. To the east, agricultural fields, orchards and croplands are found in the valleys of the semi-perennial river Sagileru. This tributary joins the Pennar River, which borders the southern part as well as a part of western side of the Sanctuary. Major floristic components of the scrub forest are thorny species of Acacia, Zizyphus and Carissa, and non-thorny species of
Cassia, Hardwickia and Anogeissus.

\section{MATERIALS AND Method}

The present work was carried out from May 2014 to April 2015 on a monthly basis. The intensive sampling area in SLWS (Image 1) was the scrub forest, which spreads over four patches (marked $A$ to $D$ on Image 1) of approximately $36 \mathrm{~km}^{2}$ (Jeganathan et al. 2005). The observations were carried out by using binoculars (10x42) during 06:00-10:00 hr \& 16:00-19:00 hr, and the identification of species was done with the help of Grimmett et al. (2011). The birds were recorded according to their status (resident, if the species was found to be present throughout the year in the locality, or local migrant, if the species was not found to be present throughout the year in the locality), abundance (estimated based on frequency of sightings of the birds: $\geq 20$ individuals of a species sighted during all visits were categorized as fairly common, $<20$ individuals of a species sighted during most of the visits were categorized as common, and birds sighted occasionally in fewer numbers between 1-5 were categorized as uncommon), seasonal visits, site and habitat types. Birds are listed following Praveen et al. (2016). Along with this, extensive camera trapping study was conducted in the study area for the detection of Critically Endangered Jerdon's Courser and camera trap data were also taken into consideration while representing avian bird diversity of the study area. Birds encountered during the study were photographed using both regular DSLR camera with 55-200mm lens and infra-red motion sensor cameras (Reconyx PC 800, PC 900, PM 75).
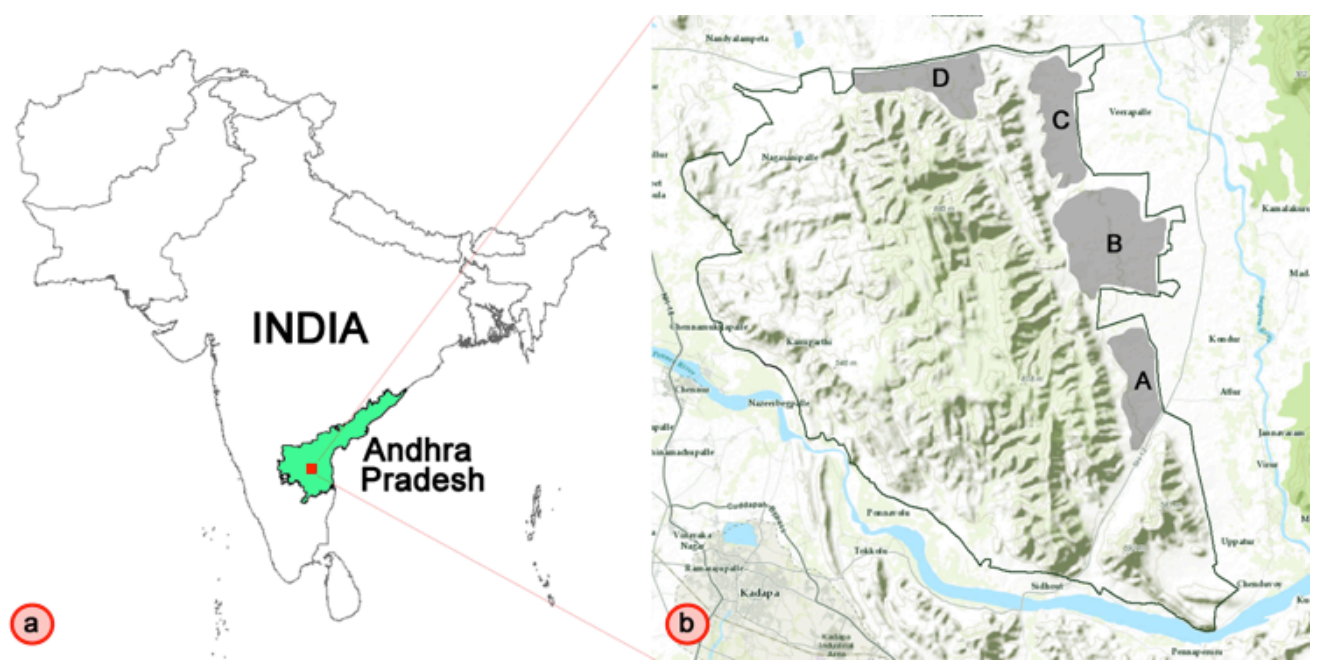

Image 1. Map of Sri Lankamalleswara Wildlife Sanctuary, Andhra Pradesh (b) showing its location in India (a) where the present study on scrubland birds was conducted between May 2014 to April 2015 (Grey shaded areas - marked A to D, are the extent of scrubland where observations were carried out) 


\section{RESULTS AND DISCUSSION}

During the present study, a total of 115 bird species belonging to 47 families and 14 orders were recorded from the scrub forest (Table 1, Images 2-67). The family Accipitridae had maximum number of bird species (8). It was followed by the families Cuculidae (7), Phasianidae (7) and Muscicapidae (6). Twenty families were represented by a single species each. Most species in the scrub forest of SLWS were resident (106 species, $92.17 \%$ ) and the rest were migratory (9 species, $7.82 \%$ ). As many as 15 are fairly common (FC), 81 species are common (C) and 19 are uncommon (UC) (Fig. 1). The only threatatned species recorded was the Painted Stork, which is a Near Threatened species.

Sampling was conducted only in the scrub forest and not in the dense forest to understand the use of scrub forest by avifauna. The scrub forest of SLWS is possibly the last known home for the Critically Endangered Jerdon's Courser and the last confirmed sighting of the species was recorded in August 2009 (BirdLife International 2009). Despite thorough surveys and deployment of 60 camera traps from 2010 to 2012, the Jerdon's Courser had not been detected (Chavan \& Barber 2012). During the present study the Jerdon's Courser was not detected. Currently, large scale research is under progress in collaboration with Andhra Pradesh Forest Department to detect the species by using advanced infra-red camera trap. This species is suspected to have declined at an unquantified rate over the last 10 years, owing to the Telugu-Ganga canal, additional habitat loss and degradation outside SLWS as a result of biotic pressures and livestock grazing.

The dependence of the settlers on the area for resources, and the increase in the number of settlers, poses a serious threat to the scrub habitat through fuel-wood collection, livestock grazing, quarrying and clearance for agriculture and plantations (Jeganathan et al. 2008), and to the birds themselves through increased disturbance. Accidental and opportunistic trapping may also be an issue (Jeganathan et al. 2008).

The present study on avifauna of scrub forest of SLWS is of preliminary nature and is an effort to bring out the bird fauna of scrub forest which has suffered severe changes in its habitat quality. This study provides baseline information for future studies to understand and compare the changes that may affect the presence of other avian species from the scrub forest of SLWS. It would be interesting to conduct a long term study to understand the impact of the Telugu-Ganga Project on other avifaunal diversity and their habitats. A further study on the various behavioural aspects of residential

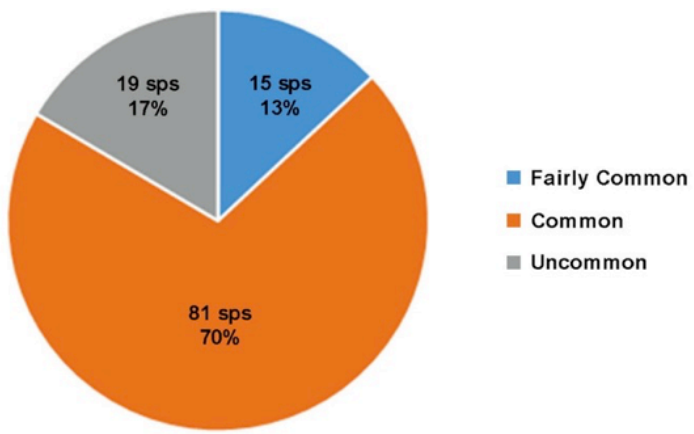

Figure 1. Abundance of the bird species in scrubland of Sri Lankamalleswara Wildlife Sanctuary, Andhra Pradesh, India

and migratory birds will enrich the fauna. Lastly, special efforts need to be taken to restore the scrub habitat for the long term conservation of Critically Endangered Jerdon's Courser, which has been recorded in the past from the current study area.

\section{REFERENCES}

BirdLife International (2009). Triple helping of good news for Jerdon's Courser. Available at: \#http://www.birdlife.org/news/ news/2009/09/jerdons_courser.html\#. (Accessed on $6^{\text {th }}$ April 2015)

Champion, F.W. \& S.K. Seth (1968). A Revised Survey of the Forest Types of India. Manager, Government of India Press, Delhi, India, 404pp.

Chavan, R. \& I. Barber (2012). In search of the elusive Jerdon's Courser: what future for one of the most endangered birds on earth? Birding ASIA 18: 102-104.

Gandhi, T. (1986). A comparative study of birds in monoculture plantations and natural scrub near Madras. MSc Thesis, University of Mumbai.

Gandhi, T. (2006). Birds in scrub jungles and monocultures in Tamil Nadu. Blackbuck 21: 1-11.

Grimmett, R., C. Inskipp \& T. Inskipp (2011). Birds of the Indian Subcontinent. Christopher Helm, London, 528pp.

Jeganathan, P., A.R. Rahmani \& R.E. Green (2005). Construction of Telugu-Ganga Canal in and around two protected areas in Cuddapah District, Andhra Pradesh, India. Immediate threat to the world population of the Critically Endangered Jerdon's Courser Rhinoptilus bitorquatus. Survey Report. Bombay Natural History Society, Mumbai, India, 10pp.

Jeganathan, P., A.R. Rahmani, R.E. Green, K. Norris, I.N. Vogiatzakis, C. Bowden \& D. Pain (2008). Quantification of threats and suggested ameliorative measures for the conservation of the Critically Endangered Jerdon's Courser Rhinoptilus bitorquatus and its habitat. Journal of the Bombay Natural History Society 105(1): 73-83.

Johnsingh, A.J.T., M.H. Martin, J. Balasingh \& V. Chelladu (1987). Vegetation and avifauna in a thorn scrub habitat in south India. Tropical Ecology 28: 22-34.

Malcolm, A. (2007). Habitat Management for Conservation: A Handbook of Techniques. Oxford University Press, Oxford, 411pp.

Praveen, J., R. Jayapal \& A. Pittie (2016). A checklist of the birds of India. Indian Birds 11(5\&6): 113-172.

Santharam, V. (1989). Species richness and avifaunal composition of scrub jungles in and around Madras City. MSc Dissertation, Pondicherry University. 
Table 1. List of birds in the scrub forest of Sri Lankamalleswara Wildlife Sanctuary, Andhra Pradesh, India

\begin{tabular}{|c|c|c|c|c|c|c|c|c|}
\hline & \multirow{2}{*}{ Common name } & \multirow{2}{*}{ Species name } & \multirow{2}{*}{ Abundance } & \multirow{2}{*}{ Status } & \multicolumn{4}{|c|}{ Scrubland patch } \\
\hline & & & & & A & B & $\mathrm{C}$ & D \\
\hline \multicolumn{9}{|c|}{ Family Ardeidae } \\
\hline 1 & Indian Pond Heron & Ardeola grayii & UC & $\mathrm{R}$ & - & + & - & - \\
\hline 2 & Cattle Egret & Bubulcus ibis & C & $\mathrm{R}$ & + & + & + & + \\
\hline \multicolumn{9}{|c|}{ Family Ciconidae } \\
\hline 3 & Painted Stork & Mycteria leucocephala & UC & $\mathrm{R}$ & - & + & - & - \\
\hline \multicolumn{9}{|c|}{ Family Threskiornithidae } \\
\hline 4 & Indian Black ibis & Pseudibis papillosa & UC & $\mathrm{R}$ & - & + & - & - \\
\hline \multicolumn{9}{|c|}{ Family Accipitridae } \\
\hline 5 & Shikra & Accipiter badius & C & $\mathrm{R}$ & + & + & + & + \\
\hline 6 & Tawny Eagle & Aquila rapax & UC & $\mathrm{R}$ & - & - & + & - \\
\hline 7 & Black-winged Kite & Elanus caeruleus & FC & $\mathrm{R}$ & - & - & + & - \\
\hline 8 & Common Kestrel & Falco tinnunculus & UC & LM & + & - & - & - \\
\hline 9 & Black Kite & Milvus migrans & UC & $\mathrm{R}$ & - & - & + & - \\
\hline 10 & Short-toed Snake Eagle & Circaetus gallicus & UC & $\mathrm{R}$ & - & - & + & - \\
\hline 11 & White-eyed buzzard & Butastur teesa & UC & $\mathrm{R}$ & + & - & - & - \\
\hline 12 & Changeable Hawk Eagle & Nisaetus cirrhatus & UC & $\mathrm{R}$ & - & - & + & - \\
\hline \multicolumn{9}{|c|}{ Family Phasianidae } \\
\hline 13 & Jungle Bush Quail & Perdicula asiatica & c & $\mathrm{R}$ & + & + & + & + \\
\hline 14 & Painted Francolin & Francolinus pictus & FC & $\mathrm{R}$ & - & + & - & - \\
\hline 15 & Grey Francolin & Francolinus pondicerianus & c & $\mathrm{R}$ & + & + & + & + \\
\hline 16 & Grey Junglefowl & Gallus sonneratii & C & $\mathrm{R}$ & + & + & + & + \\
\hline 17 & Indian Peafowl & Pavo cristatus & C & $\mathrm{R}$ & + & + & + & + \\
\hline 18 & Red Spurfowl & Galloperdix spadicea & C & $\mathrm{R}$ & + & + & + & + \\
\hline 19 & Painted Spurfowl & Galloperdix lunulata & UC & $\mathrm{R}$ & + & - & - & - \\
\hline \multicolumn{9}{|c|}{ Family Turnicidae } \\
\hline 20 & Yellow-legged Buttonquail & Turnix tanki & c & $\mathrm{R}$ & + & + & + & + \\
\hline 21 & Barred Buttonquail & Turnix suscitator & C & $\mathrm{R}$ & + & + & + & + \\
\hline \multicolumn{9}{|c|}{ Family Rallidae } \\
\hline 22 & White-breasted Waterhen & Amaurornis phoenicurus & UC & $\mathrm{R}$ & - & + & - & - \\
\hline \multicolumn{9}{|c|}{ Family Burhinidae } \\
\hline 23 & Indian Thick Knee & Burhinus oedicnemus & C & $\mathrm{R}$ & + & + & + & + \\
\hline \multicolumn{9}{|c|}{ Family Glareolidae } \\
\hline 24 & Indian Courser & Cursorius coromandelicus & UC & $\mathrm{R}$ & - & + & - & - \\
\hline \multicolumn{9}{|c|}{ Family Charadridae } \\
\hline 25 & Red-wattled Lapwing & Vanellus indicus & C & $\mathrm{R}$ & + & + & + & + \\
\hline 26 & Yellow-wattled Lapwing & Vanellus malabaricus & C & $\mathrm{R}$ & + & + & + & + \\
\hline \multicolumn{9}{|c|}{ Family Columbidae } \\
\hline 27 & $\begin{array}{l}\text { Chestnut-bellied } \\
\text { Sandgrouse }\end{array}$ & Pterocles exustus & C & $\mathrm{R}$ & + & + & + & + \\
\hline 28 & Painted Sandgrouse & Pterocles indicus & C & $\mathrm{R}$ & + & + & + & + \\
\hline 29 & Eurasian Collared Dove & Streptopelia decaocto & C & $\mathrm{R}$ & + & + & + & + \\
\hline 30 & Laughing Dove & Streptopelia senegalensis & C & $\mathrm{R}$ & + & + & + & + \\
\hline 31 & Spotted Dove & Streptopelia chinensis & C & $\mathrm{R}$ & + & + & + & + \\
\hline
\end{tabular}




\begin{tabular}{|c|c|c|c|c|c|c|c|c|}
\hline & \multirow{2}{*}{ Common name } & \multirow{2}{*}{ Species name } & \multirow{2}{*}{ Abundance } & \multirow{2}{*}{ Status } & \multicolumn{4}{|c|}{ Scrubland patch } \\
\hline & & & & & $A$ & B & C & D \\
\hline \multicolumn{9}{|c|}{ Family Psittacidae } \\
\hline 32 & Rose-ringed Parakeet & Psittacula krameri & C & $\mathrm{R}$ & + & + & + & + \\
\hline \multicolumn{9}{|c|}{ Family Cuculidae } \\
\hline 33 & Greater Coucal & Centropus sinensis & C & $\mathrm{R}$ & + & + & + & + \\
\hline 34 & Pied Cuckoo & Clamator jacobinus & C & LM & - & + & + & + \\
\hline 35 & Asian Koel & Eudynamyus scolopacea & c & $\mathrm{R}$ & + & + & + & + \\
\hline 36 & Common Hawk-Cuckoo & Heirococcyx varius & c & $R$ & + & + & + & + \\
\hline 37 & Blue-faced Malkoha & $\begin{array}{l}\text { Phaenicophaeus } \\
\text { viridirostris }\end{array}$ & C & $\mathrm{R}$ & + & + & + & + \\
\hline 38 & Drongo Cuckoo & Sumiculus lugubris & C & $\mathrm{R}$ & + & + & + & + \\
\hline 39 & Sirkeer Malkoha & Taccocua leschenaultii & C & $R$ & + & + & + & + \\
\hline \multicolumn{9}{|c|}{ Family Strigidae } \\
\hline 40 & Spotted Owlet & Athene brama & c & $R$ & + & + & + & + \\
\hline 41 & Indian Eagle Owl & Bubo bengalensis & UC & $\mathrm{R}$ & - & - & + & - \\
\hline \multicolumn{9}{|c|}{ Family Caprimulgidae } \\
\hline 42 & Common Indian Nightjar & Caprimulgus asiaticus & C & $R$ & + & + & + & + \\
\hline 43 & Jerdon's Nightjar & Caprimulgus atripennis & C & $\mathrm{R}$ & + & + & + & + \\
\hline 44 & Savanna Nightjar & Caprimulgus affinis & c & $\mathrm{R}$ & + & + & + & + \\
\hline \multicolumn{9}{|c|}{ Family Apopidae } \\
\hline 45 & Asian Palm Swift & Cypsiurus balasiensis & FC & $\mathrm{R}$ & + & + & + & + \\
\hline \multicolumn{9}{|c|}{ Family Alcedinidae } \\
\hline 46 & White-throated Kingfisher & Halcyon smyrnensis & FC & $\mathrm{R}$ & - & - & + & - \\
\hline \multicolumn{9}{|c|}{ Family Meropidae } \\
\hline 47 & Green Bee-eater & Merops orientalis & c & $\mathrm{R}$ & + & + & + & + \\
\hline 48 & Blue-tailed Bee-eater & Merops philippinus & FC & LM & + & + & - & - \\
\hline \multicolumn{9}{|c|}{ Family Coraciidae } \\
\hline 49 & Indian Roller & Coracias benghalensis & C & $\mathrm{R}$ & + & + & + & + \\
\hline \multicolumn{9}{|c|}{ Family Upupidae } \\
\hline 50 & Common Hoopoe & Upupa epops & C & $\mathrm{R}$ & + & + & + & + \\
\hline \multicolumn{9}{|c|}{ Family Bucerotidae } \\
\hline 51 & Indian Grey Hornbill & Ocyceros birostris & C & $\mathrm{R}$ & + & + & + & + \\
\hline \multicolumn{9}{|c|}{ Family Picidae } \\
\hline 52 & $\begin{array}{l}\text { Black-rumped Flamebacked } \\
\text { Woodpecker }\end{array}$ & Dinopium benghalense & C & $\mathrm{R}$ & - & - & + & + \\
\hline 53 & Eurasian Wryneck & Jynx torquilla & UC & LM & - & - & + & - \\
\hline \multicolumn{9}{|c|}{ Family Pittidae } \\
\hline 54 & Indian Pitta & Pitta brachyura & FC & R & + & + & + & + \\
\hline \multicolumn{9}{|c|}{ Family Alaudidae } \\
\hline 55 & Ashy-Crowned Sparrow Lark & Eremopterix grisea & c & $\mathrm{R}$ & + & + & + & + \\
\hline 56 & Indian Bushlark & Mirafra erythroptera & C & $\mathrm{R}$ & + & + & + & + \\
\hline 57 & Jerdon's Bushlark & Mirafra affinis & C & $R$ & + & + & + & + \\
\hline 58 & Rufous-tailed Lark & Ammomanes phoenicura & c & $\mathrm{R}$ & + & + & + & + \\
\hline \multicolumn{9}{|c|}{ Family Hirundinidae } \\
\hline 59 & Barn Swallow & Hirundo rustica & C & R & + & + & + & + \\
\hline
\end{tabular}




\begin{tabular}{|c|c|c|c|c|c|c|c|c|}
\hline & \multirow{2}{*}{ Common name } & \multirow{2}{*}{ Species name } & \multirow{2}{*}{ Abundance } & \multirow{2}{*}{ Status } & \multicolumn{4}{|c|}{ Scrubland patch } \\
\hline & & & & & A & B & C & D \\
\hline \multicolumn{9}{|c|}{ Family Motacillidae } \\
\hline 60 & Paddy-field pipit & Anthus rufulus & C & $\mathrm{R}$ & + & + & + & + \\
\hline 61 & White-browed wagtail & Motacilla maderaspatensis & UC & $\mathrm{R}$ & - & - & + & - \\
\hline \multicolumn{9}{|c|}{ Family Campephagidae } \\
\hline 62 & Black-headed Cuckoo-shrike & Coracina melanoptera & FC & $\mathrm{R}$ & - & - & + & - \\
\hline 63 & Small minivet & Pericrocotus cinnamomeus & C & $\mathrm{R}$ & + & + & + & + \\
\hline \multicolumn{9}{|c|}{ Family Prionopidae } \\
\hline 64 & Common Woodshrike & Tephrodornis pondicerianus & C & $\mathrm{R}$ & + & + & + & + \\
\hline \multicolumn{9}{|c|}{ Family Monarchidae } \\
\hline 65 & India Paradise-flycatcher & Trepsiphone paradisi & FC & $\mathrm{R}$ & + & + & + & + \\
\hline \multicolumn{9}{|c|}{ Family Rhipiduridae } \\
\hline 66 & White-browed Fantail & Rhipidura aureola & C & $\mathrm{R}$ & + & + & + & + \\
\hline \multicolumn{9}{|c|}{ Family Pycnonotidae } \\
\hline 67 & Red-vented Bulbul & Pycnonotus cafer & C & $\mathrm{R}$ & + & + & + & + \\
\hline 68 & Red-whiskered Bulbul & Pycnonotus jocosus & C & $\mathrm{R}$ & + & + & + & + \\
\hline 69 & White-browed Bulbul & Pycnonotus luteolus & C & $\mathrm{R}$ & + & + & + & + \\
\hline \multicolumn{9}{|c|}{ Family Aegithinidae } \\
\hline 70 & Common lora & Aegithina tiphia & C & $\mathrm{R}$ & + & + & + & + \\
\hline \multicolumn{9}{|c|}{ Family Lanidae } \\
\hline 71 & Bay-backed Shrike & Lanius vittatus & C & $\mathrm{R}$ & + & + & + & + \\
\hline 72 & Southern Grey Shrike & Lanius meridionalis & C & $\mathrm{R}$ & + & - & + & - \\
\hline 73 & Long-tailed Shrike & Lanius schach & C & $\mathrm{R}$ & + & - & + & - \\
\hline \multicolumn{9}{|c|}{ Family Muscicapidae } \\
\hline 74 & Oriental Magpie-Robin & Copsychus saularis & C & $\mathrm{R}$ & + & + & + & + \\
\hline 75 & Tickell's Blue Flycatcher & Cyornis tickelliae & UC & $\mathrm{R}$ & + & - & - & - \\
\hline 76 & Pied Bushchat & Saxicola caprata & FC & $\mathrm{R}$ & + & + & + & + \\
\hline 77 & Common Stonechat & Saxicola torquata & C & $\mathrm{R}$ & + & - & + & - \\
\hline 78 & Indian Robin & Saxicoloides fulicata & C & $\mathrm{R}$ & + & + & + & + \\
\hline 79 & Asian Brown Flycatcher & Muscicapa dauurica & UC & LM & + & - & - & - \\
\hline \multicolumn{9}{|c|}{ Family Timaliidae } \\
\hline 80 & Jungle Babbler & Turdoides striatus & C & $\mathrm{R}$ & + & + & + & + \\
\hline 81 & Large Grey Babbler & Turdoides malcolmi & C & $\mathrm{R}$ & + & + & + & + \\
\hline 82 & Rufous Babbler & Turdoides subrufus & C & $\mathrm{R}$ & + & + & + & + \\
\hline 83 & Yellow-billed Babbler & Turdoides affinis & C & $\mathrm{R}$ & + & + & + & + \\
\hline 84 & Yellow-eyed Babbler & Chrysomma sinense & FC & $\mathrm{R}$ & - & - & + & - \\
\hline 85 & Tawny-bellied Babbler & Dumetia hyperythra & FC & $\mathrm{R}$ & - & - & + & - \\
\hline \multicolumn{9}{|c|}{ Family Cisticolidae } \\
\hline 86 & Ashy Prinia & Prinia socialis & C & $\mathrm{R}$ & + & + & + & + \\
\hline 87 & Jungle Prinia & Prinia sylvatica & C & $\mathrm{R}$ & + & + & + & + \\
\hline 88 & Plain Prinia & Prinia inornata & C & $\mathrm{R}$ & + & + & + & + \\
\hline 89 & Grey-breasted Prinia & Prinia hodgsonii & C & $\mathrm{R}$ & + & + & + & + \\
\hline 90 & Ziting Cisticola & Cisticola juncidis & UC & $\mathrm{R}$ & - & - & + & - \\
\hline \multicolumn{9}{|c|}{ Family Sylviidae } \\
\hline 91 & Blyth's Reed Warbler & Acrocephalus dumetorum & FC & LM & - & + & - & - \\
\hline
\end{tabular}




\begin{tabular}{|c|c|c|c|c|c|c|c|c|}
\hline & \multirow{2}{*}{ Common name } & \multirow{2}{*}{ Species name } & \multirow{2}{*}{ Abundance } & \multirow{2}{*}{ Status } & \multicolumn{4}{|c|}{ Scrubland patch } \\
\hline & & & & & A & B & C & D \\
\hline 92 & Booted Warbler & Iduna caligata & FC & LM & - & + & - & - \\
\hline 93 & Common Tailorbird & Orthotomus sutorius & c & R & + & + & + & + \\
\hline 94 & Hume's Whitethroat & Sylvia althaea & C & $\mathrm{R}$ & + & + & + & + \\
\hline \multicolumn{9}{|c|}{ Family Nectarinidae } \\
\hline 95 & Purple Sunbird & Cinnyris asiaticus & C & $\mathrm{R}$ & + & + & + & + \\
\hline 96 & Purple-rumped Sunbird & Leptocoma zeylonica & C & $\mathrm{R}$ & + & + & + & + \\
\hline \multicolumn{9}{|c|}{ Family Dicaeidae } \\
\hline 97 & Pale-billed Flowerpecker & Dicaeum erythrorhynchos & C & $\mathrm{R}$ & + & + & + & + \\
\hline \multicolumn{9}{|c|}{ Family Oriolidae } \\
\hline 98 & Indian Golden Oriole & Oriolus kundoo & UC & LM & + & + & + & + \\
\hline 99 & Black-hooded Oriole & Oriolus xanthornus & UC & $\mathrm{R}$ & + & + & + & + \\
\hline \multicolumn{9}{|c|}{ Family Dicruridae } \\
\hline 100 & Black Drongo & Dicrurus macrocercus & C & $\mathrm{R}$ & + & + & + & + \\
\hline 101 & Ashy Drongo & Dicrurus leucophaeus & c & $\mathrm{R}$ & + & + & + & + \\
\hline 102 & White-bellied Drongo & Dicrurus caerulescens & C & $\mathrm{R}$ & + & + & + & + \\
\hline \multicolumn{9}{|c|}{ Family Artamidae } \\
\hline 103 & Ashy Woodswallow & Artamus fuscus & C & $\mathrm{R}$ & + & + & + & + \\
\hline \multicolumn{9}{|c|}{ Family Corvidae } \\
\hline 104 & Jungle Crow & Corvus splendens & C & $\mathrm{R}$ & + & + & + & + \\
\hline 105 & House Crow & Corvus macrorhynchos & C & $\mathrm{R}$ & + & + & + & + \\
\hline 106 & Rufous Treepie & Dendrocitta vagabunda & C & $\mathrm{R}$ & + & + & + & + \\
\hline \multicolumn{9}{|c|}{ Family Sturnidae } \\
\hline 107 & Common Myna & Acridotheres tristis & C & $\mathrm{R}$ & + & + & + & + \\
\hline 108 & Brahminy Starling & Sturnus pagodarum & C & $\mathrm{R}$ & + & + & + & + \\
\hline 109 & Rosy Starling & Sturnus roseus & C & LM & + & + & + & - \\
\hline \multicolumn{9}{|c|}{ Family Ploceidae } \\
\hline 110 & Baya Weaver & Ploceus philippinus & C & $\mathrm{R}$ & + & + & + & + \\
\hline \multicolumn{9}{|c|}{ Family Estrildidae } \\
\hline 111 & Scaly-breasted Munia & Lonchura punctulata & C & $\mathrm{R}$ & + & + & + & + \\
\hline 112 & White-throated Munia & Lonchura malabarica & C & $\mathrm{R}$ & - & + & + & + \\
\hline 113 & Black-headed Munia & Lonchura malacca & FC & $\mathrm{R}$ & + & + & + & + \\
\hline \multicolumn{9}{|c|}{ Family Passeridae } \\
\hline 114 & House Sparrow & Passer domesticus & FC & $\mathrm{R}$ & + & + & + & + \\
\hline 115 & $\begin{array}{l}\text { Chestnut-shouldered } \\
\text { Petronia }\end{array}$ & Petronia xanthocollis & C & $\mathrm{R}$ & + & + & + & + \\
\hline
\end{tabular}

Status: R - Resident, LM - Local Migrant; Abundance: FC - Fairly common, C - Common, UC - Uncommon; '+' - Present, '-' - Absent, 


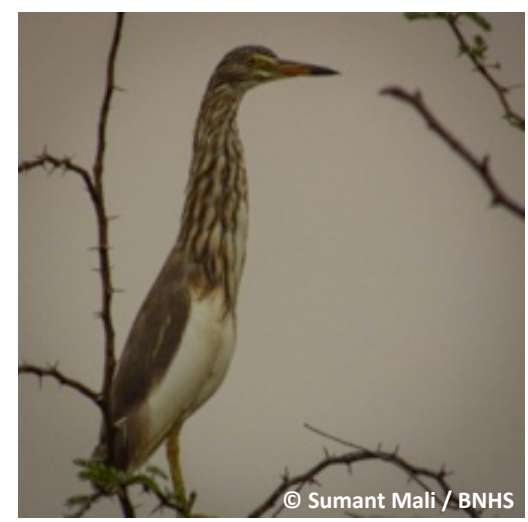

Image 2. Ardeola grayii

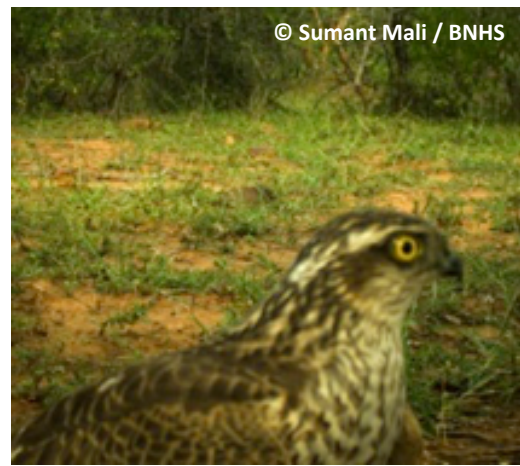

Image 5. Accipiter badius

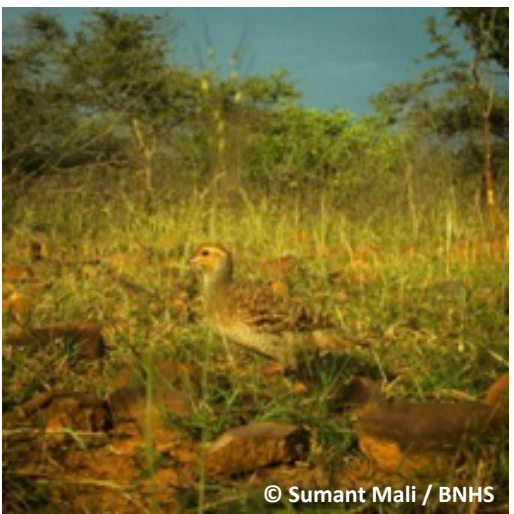

Image 8. Francolinus pondicerianus

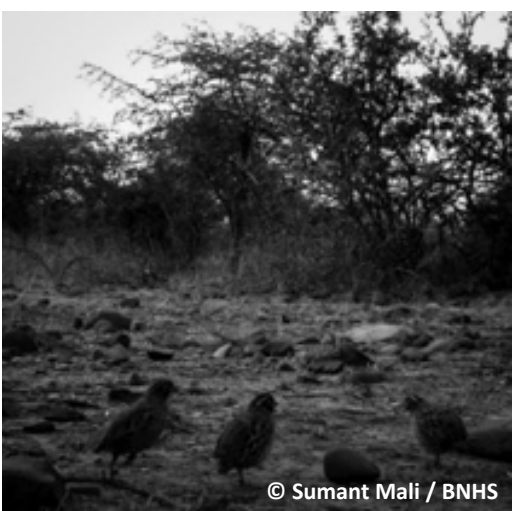

Image 11. Perdicula asiatica

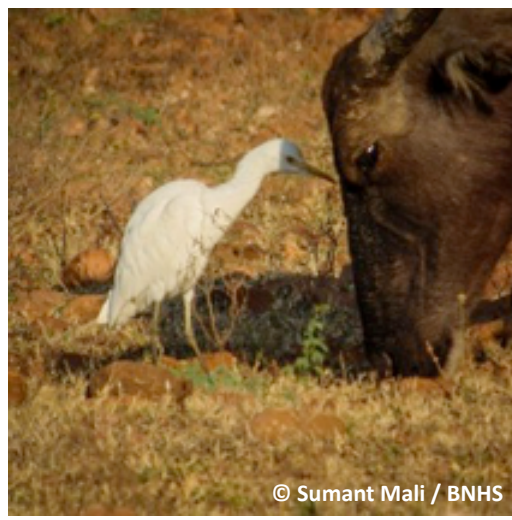

Image 3. Bubulcus ibis

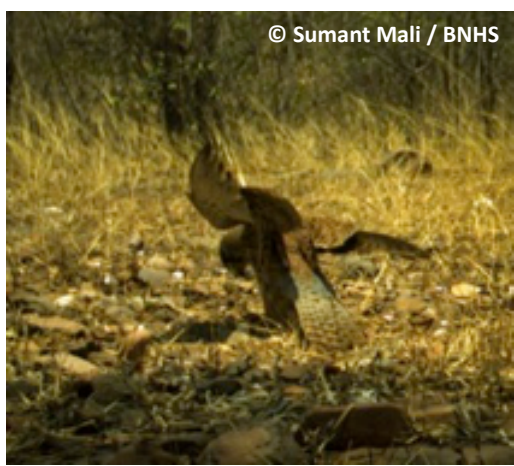

Image 6. Falco tinnunculus

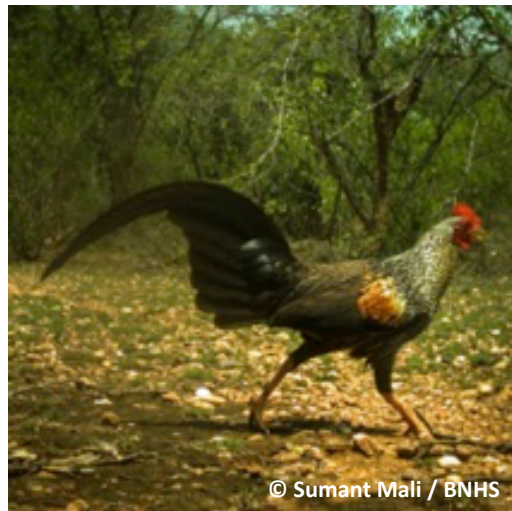

Image 9. Gallus sonneratii

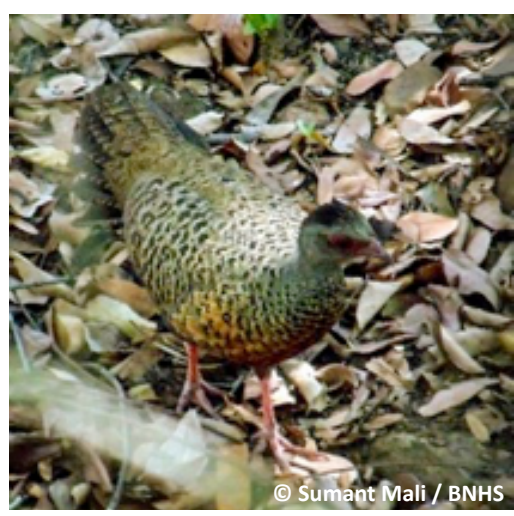

Image 12. Galloperdix spadicea

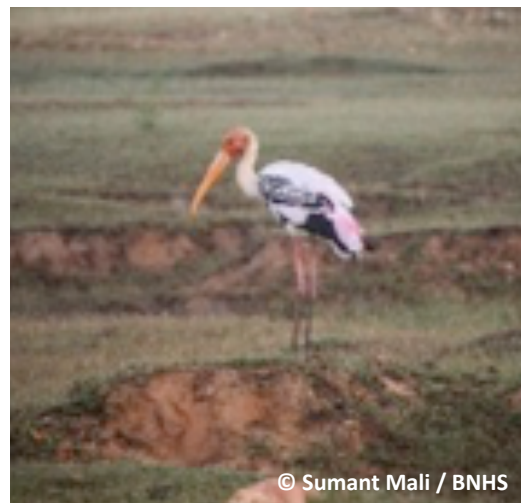

Image 4. Mycteria leucocephala

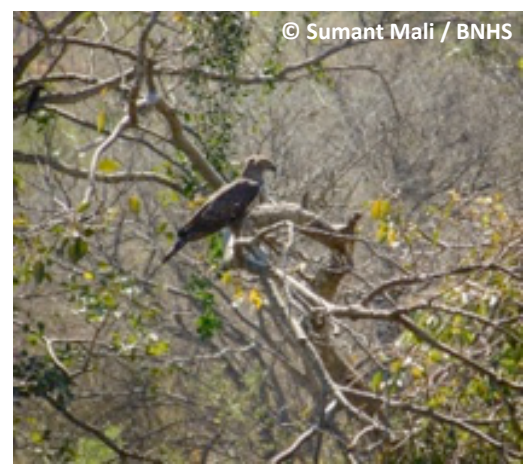

Image 7. Nisaetus cirrhatus

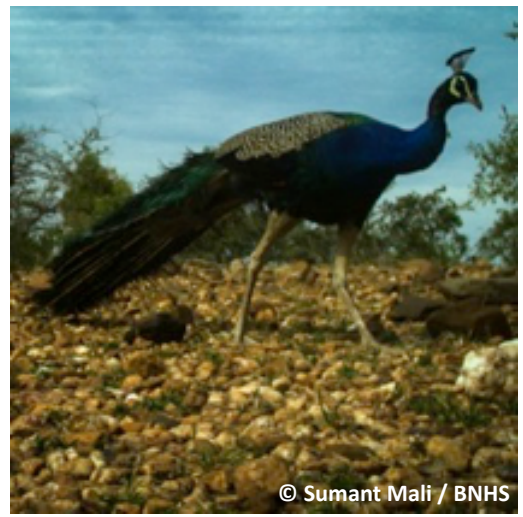

Image 10. Pavo cristatus

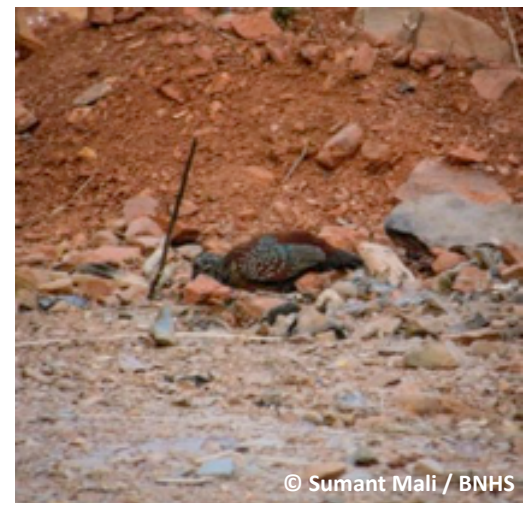

Image 13. Galloperdix lunulata 


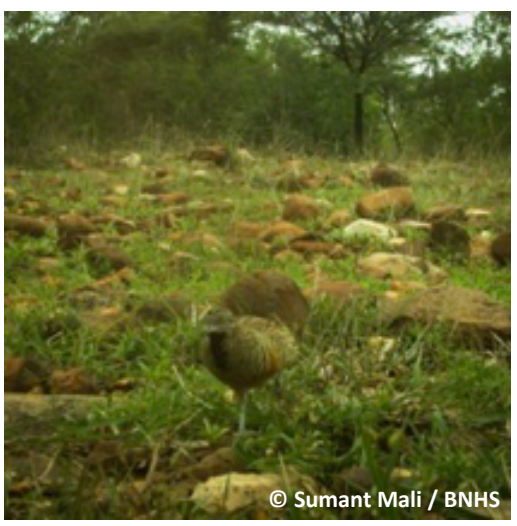

Image 14. Turnix suscitator

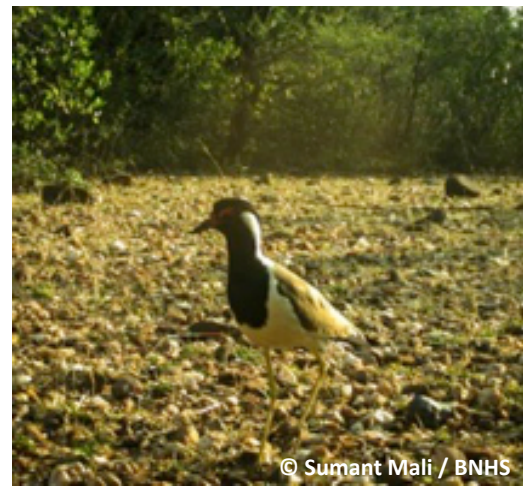

Image 17. Vanellus indicus

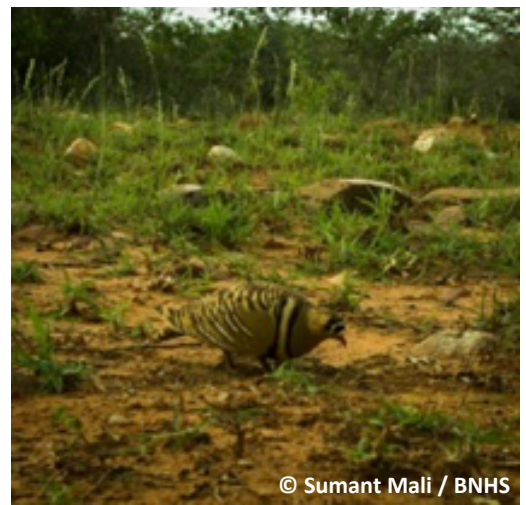

Image 20. Pterocles indicus

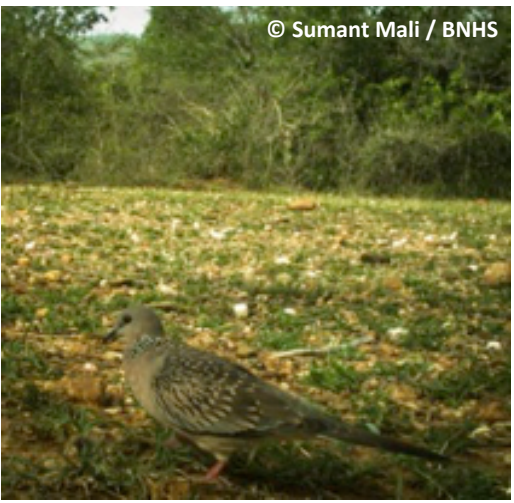

Image 23. Streptopelia chinensis

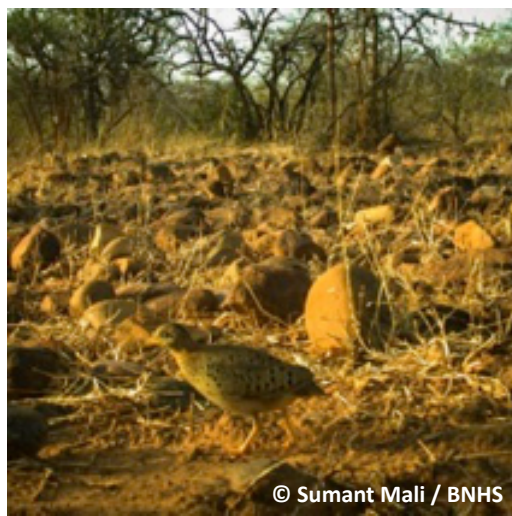

Image 15. Turnix tanki

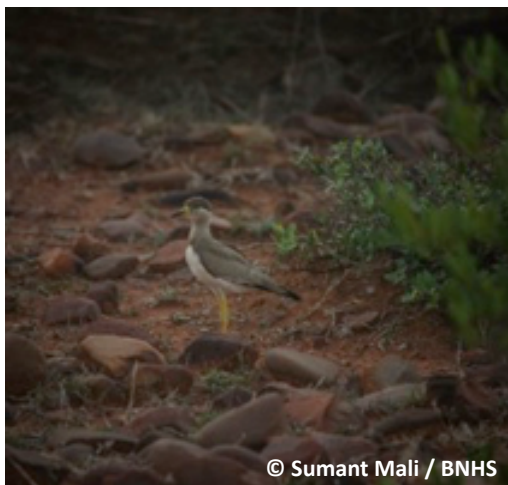

Image 18. Vanellus malabaricus

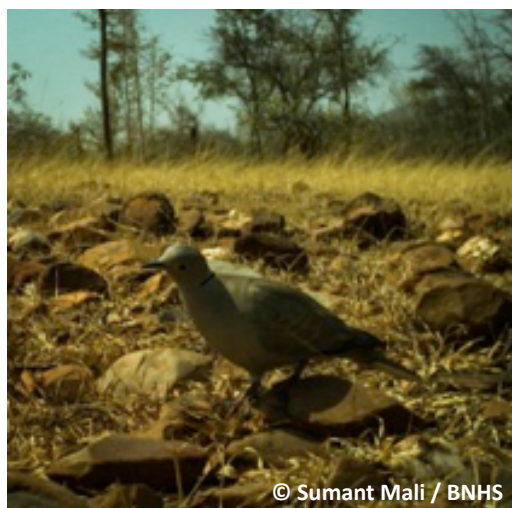

Image 21. Streptopelia decaocto

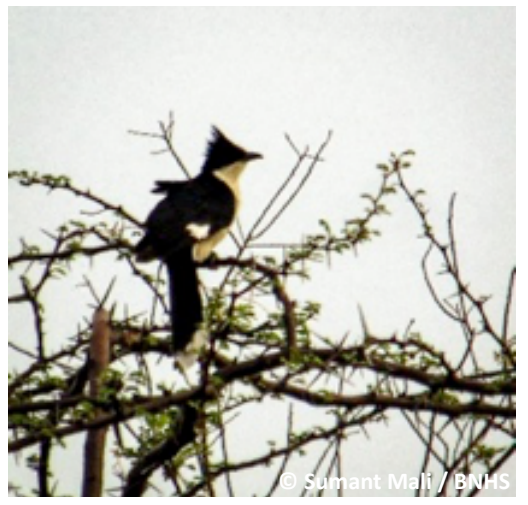

Image 24. Clamator jacobinus

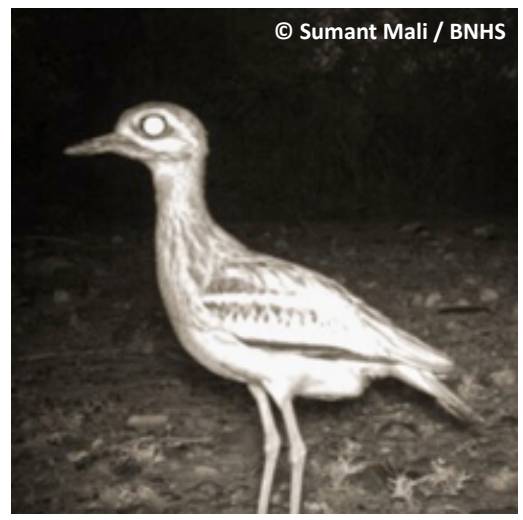

Image 16. Burhinus oedicnemus

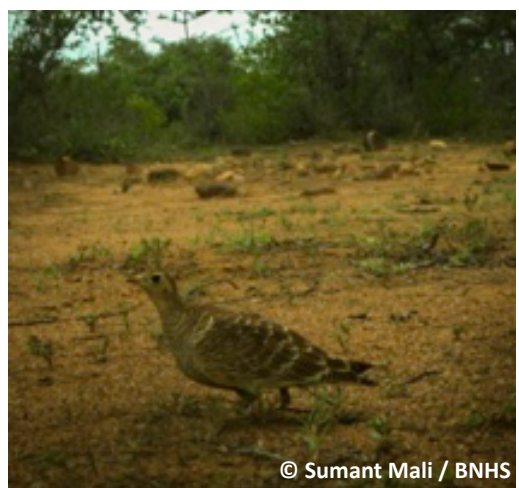

Image 19. Pterocles exustus

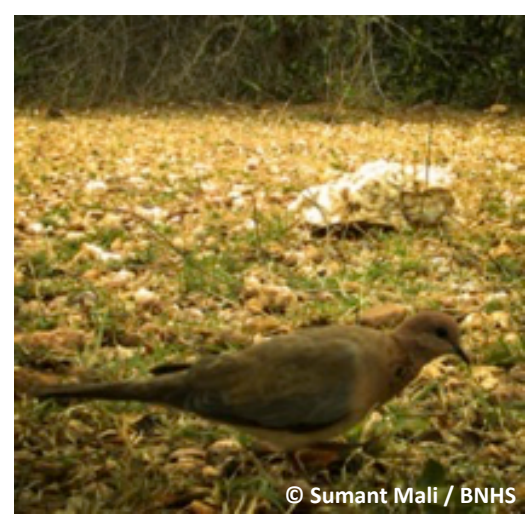

Image 22. Streptopelia senegalensis

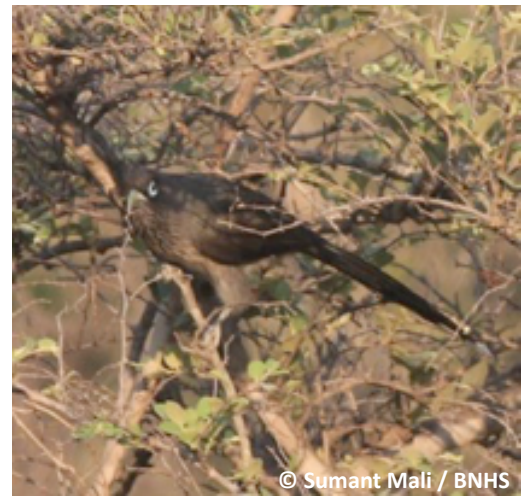

Image 25. Phaenicophaeus viridirostris 


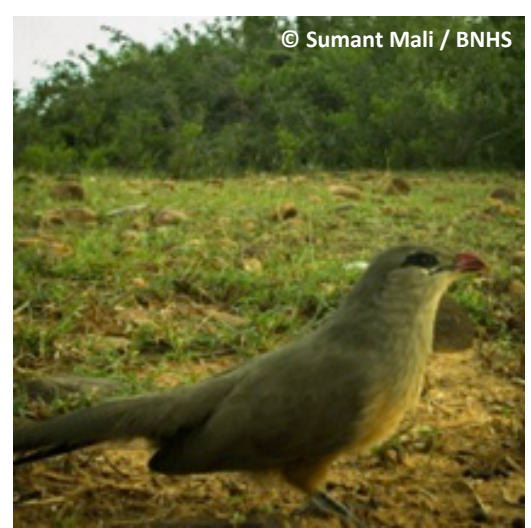

Image 26. Taccocua leschenaultii

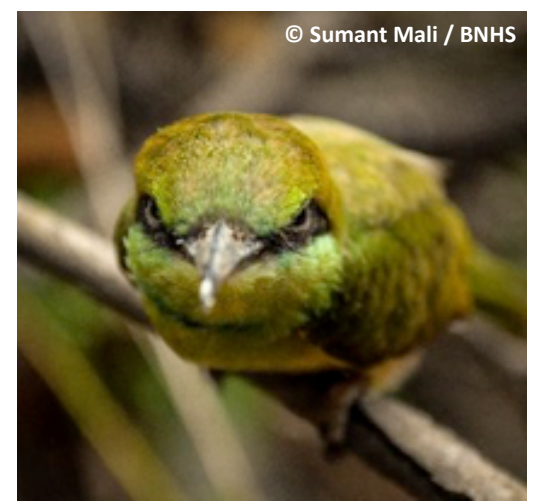

Image 29. Merops oreintalis

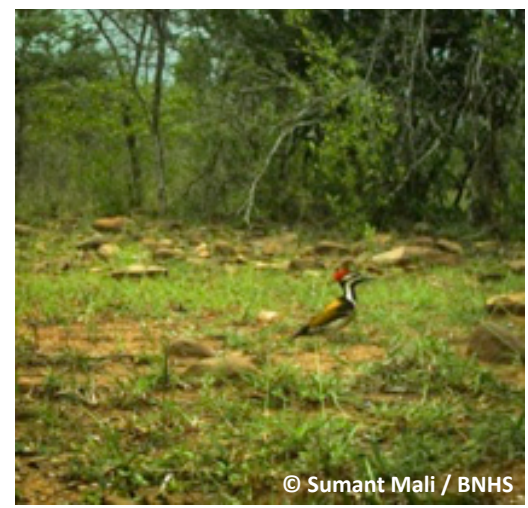

Image 32. Dinopium benghalensis

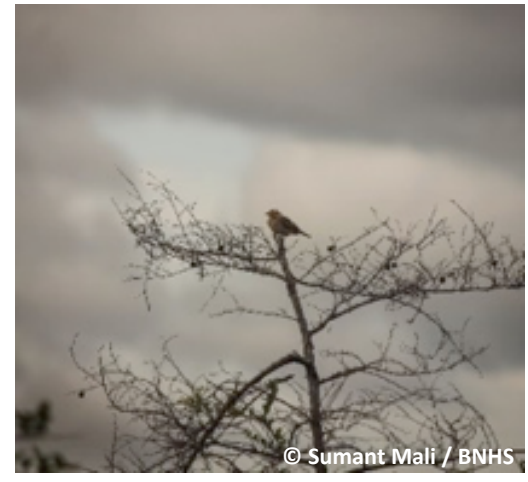

Image 35. Mirafra erythoptera

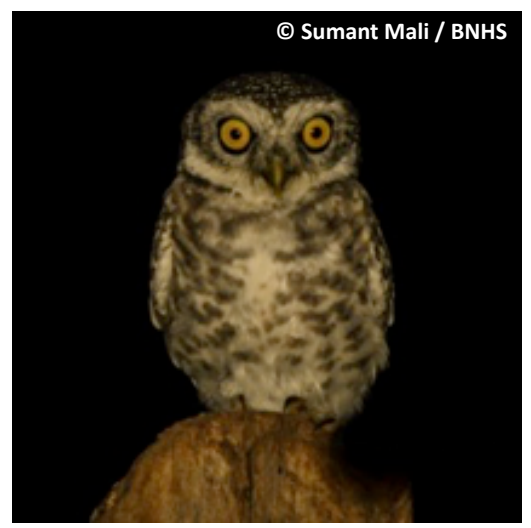

Image 27. Athene brama

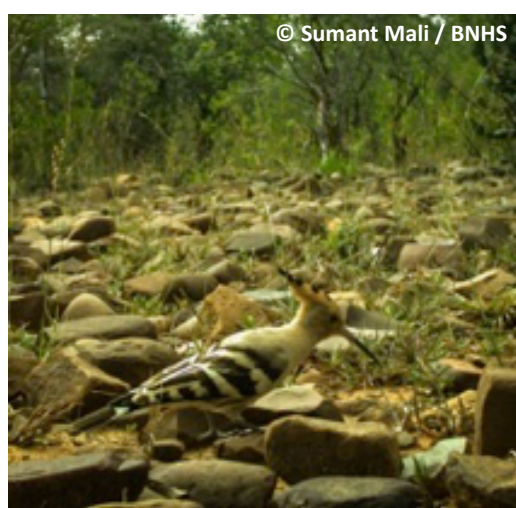

Image 30. Upupa epops

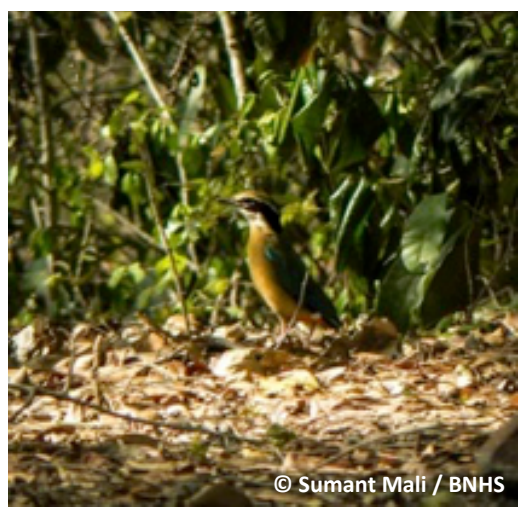

Image 33. Pitta brachyura

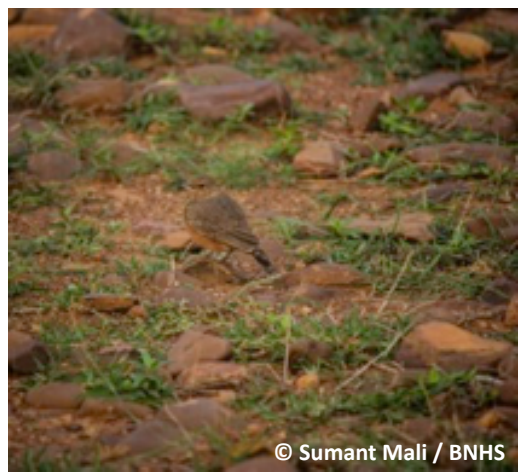

Image 36. Ammomanes phoenicura

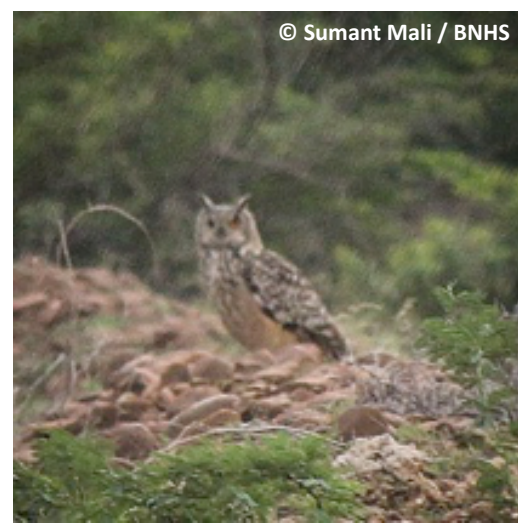

Image 28. Bubo bengalensis

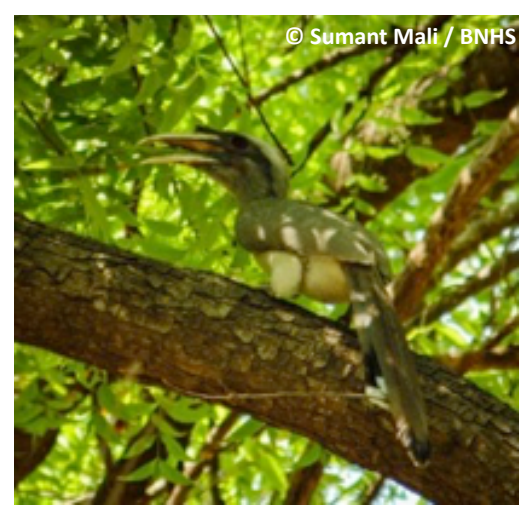

Image 31. Ocyceros birostris

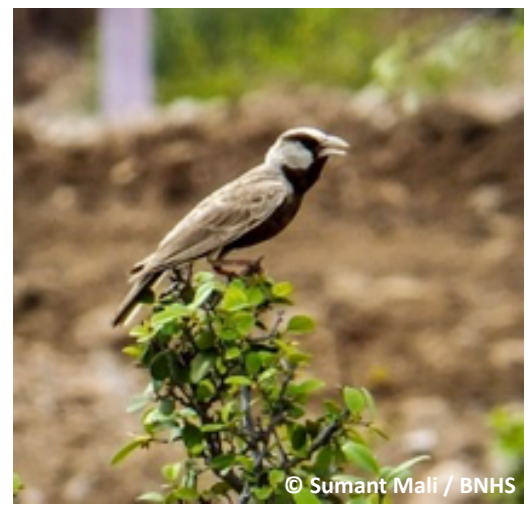

Image 34. Eremopterix grisea

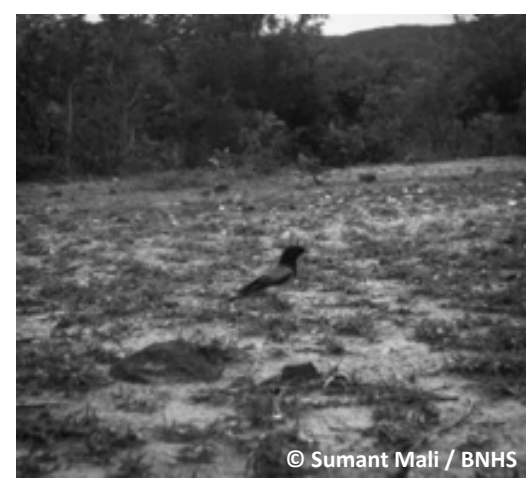

Image 37. Coracina melanoptera 


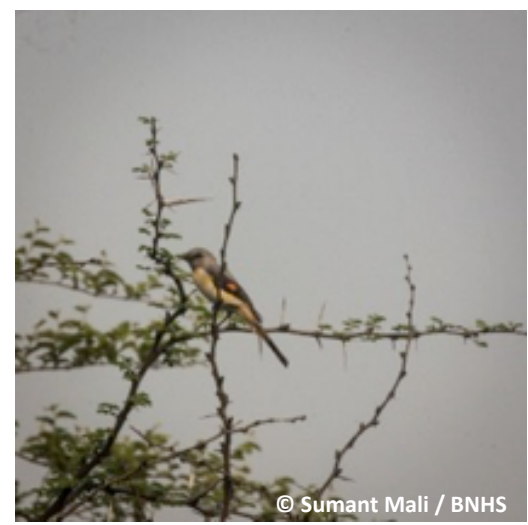

Image 38. Pericrocotus cinnamomeus

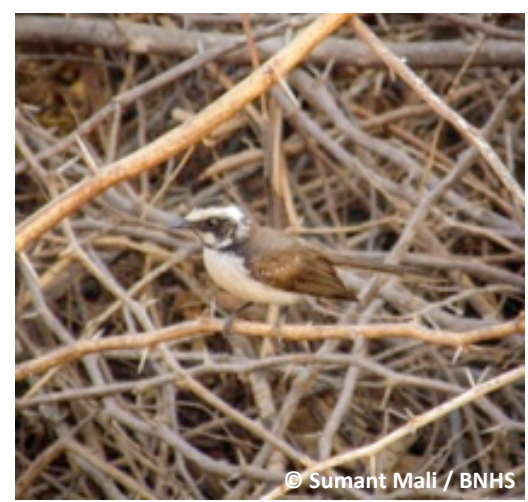

Image 41. Rhipidura aureola

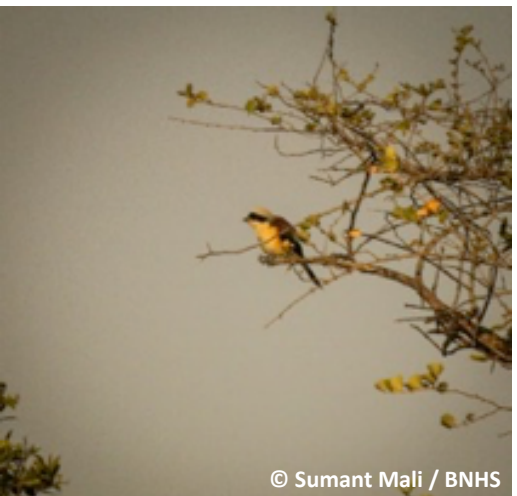

Image 44. Lanius vittatus

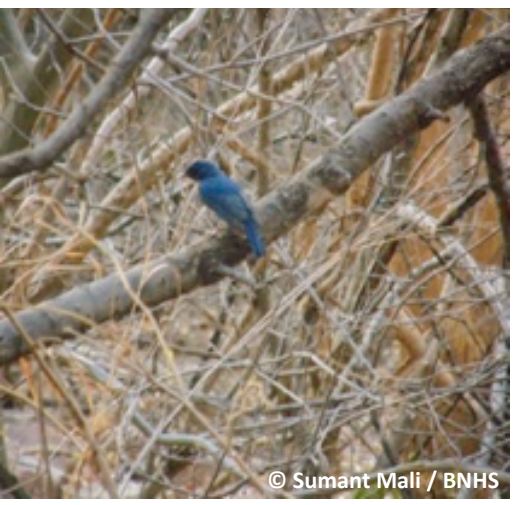

Image 47. Cyornis tickelliae

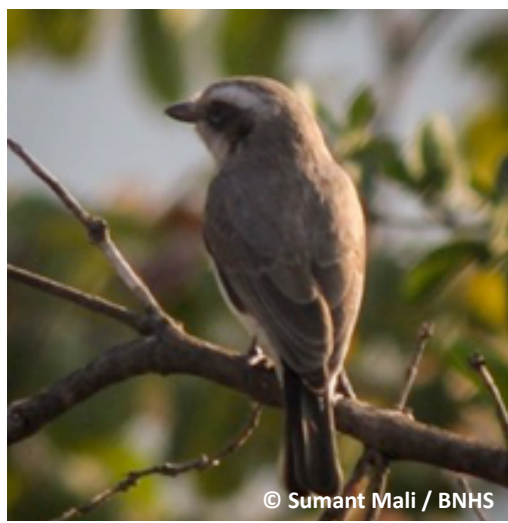

Image 39. Tephrodornis pondicerianus

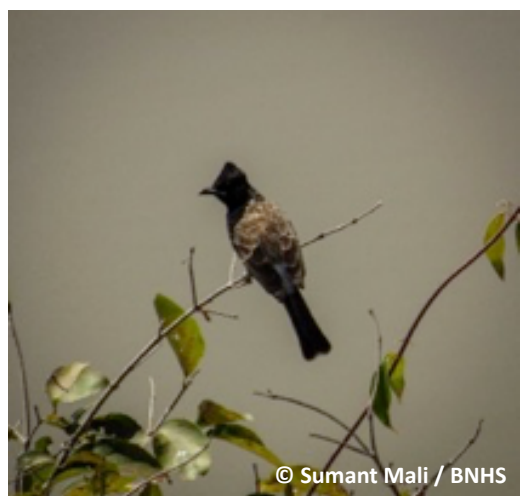

Image 42. Pycnonotus cafer

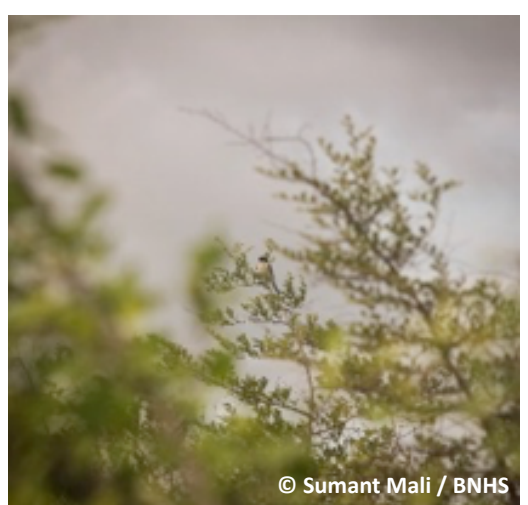

Image 45. Lanius meridionalis

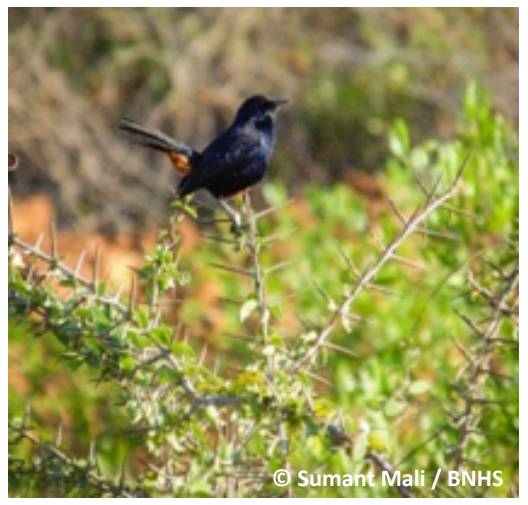

Image 48. Saxicoloides fulicata

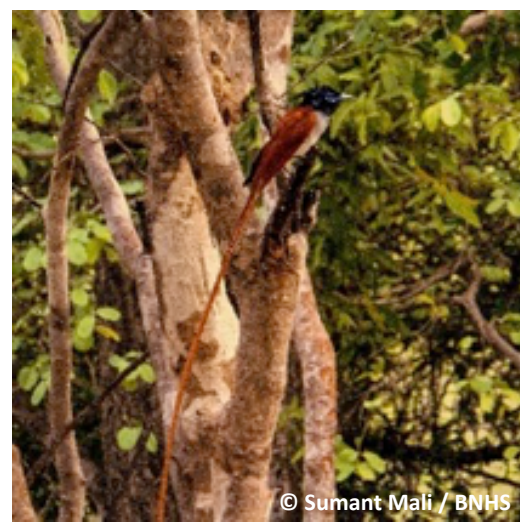

Image 40. Trepsiphone paradisi

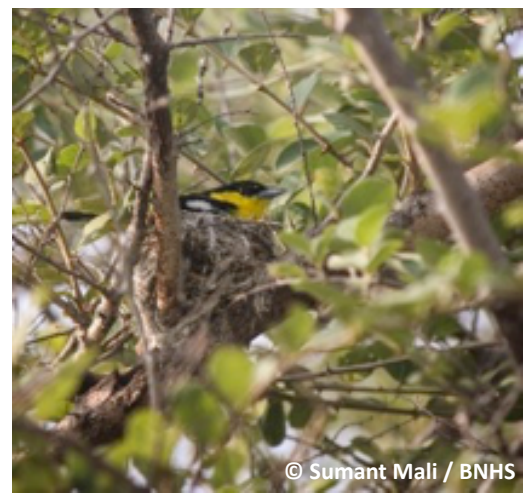

Image 43. Aegithina tiphia

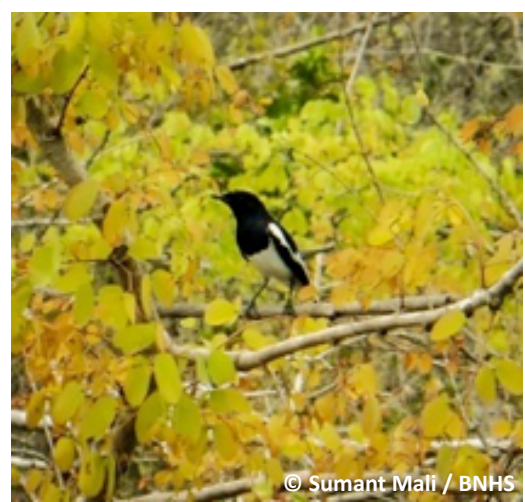

Image 46. Copsychus saularis

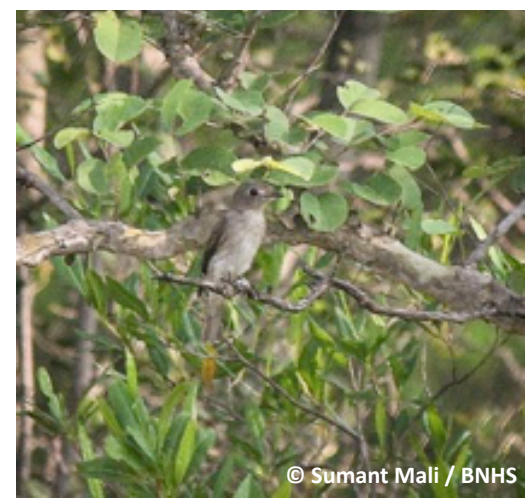

Image 49. Muscicapa dauurica 


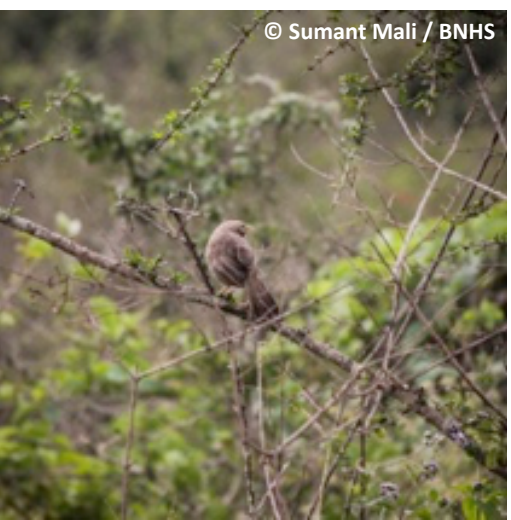

Image 50. Turdoides striatus

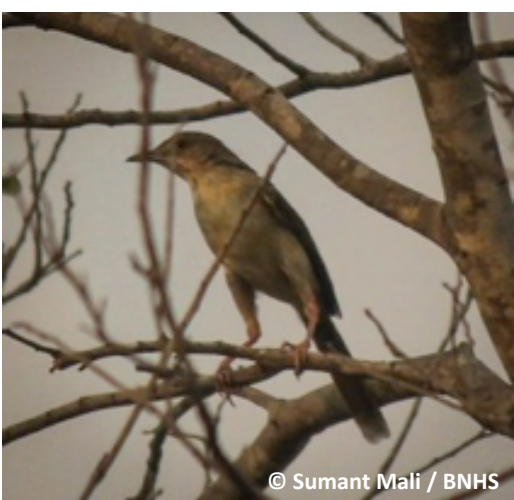

Image 53. Prinia sylvatica

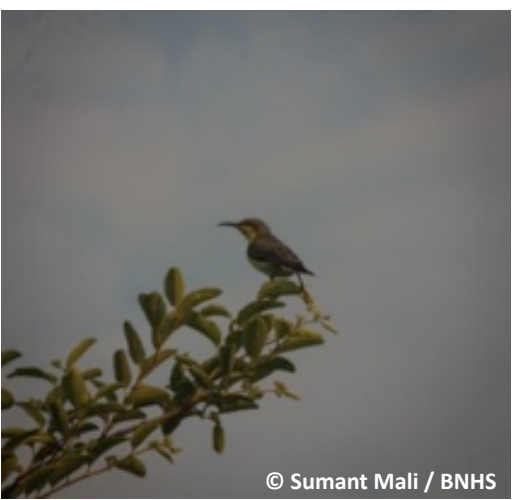

Image 56. Cinnyris asiaticus

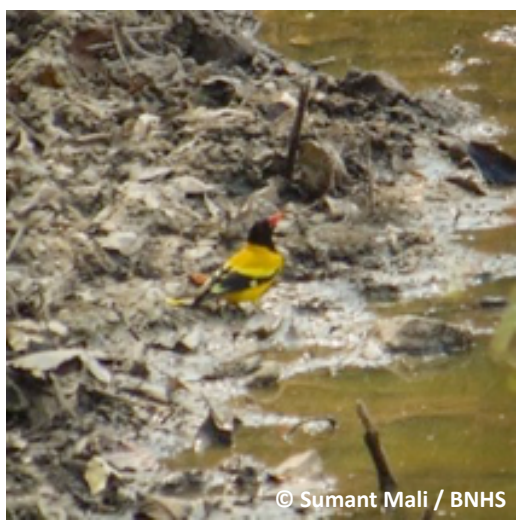

Image 59. Oriolus xanthornus

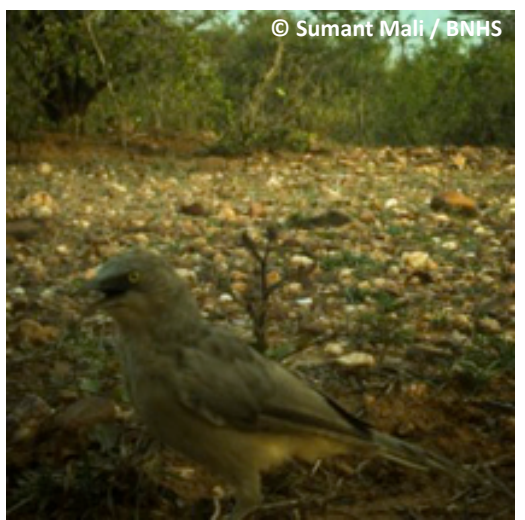

Image 51. Turdoides malcolmi

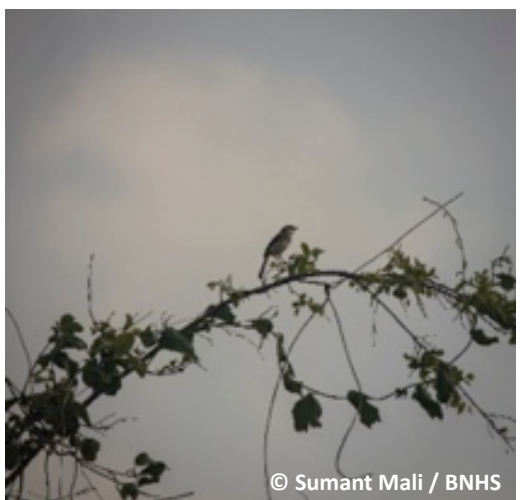

Image 54. Prinia hodgsonii

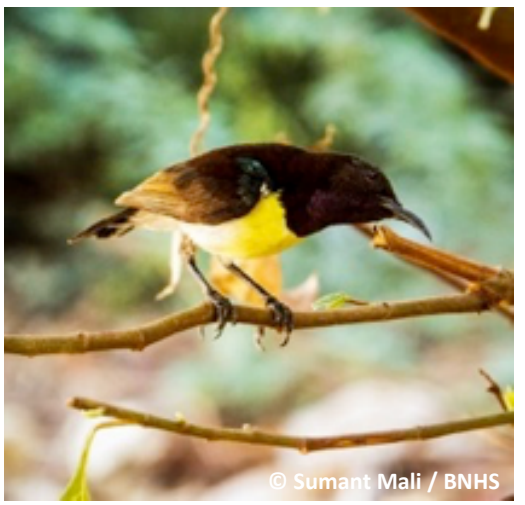

Image 57. Leptocoma zeylonica

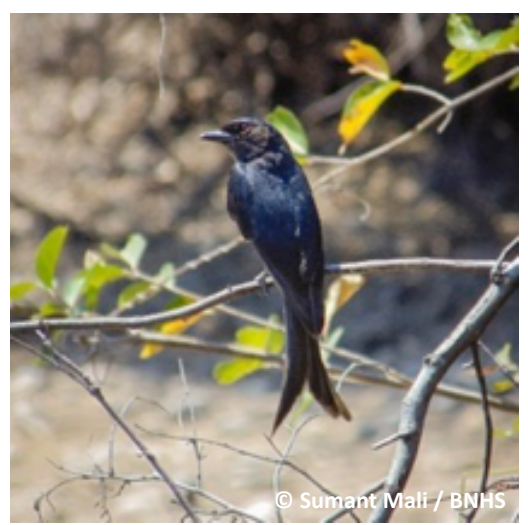

Image 60. Dicrurus macrocercus

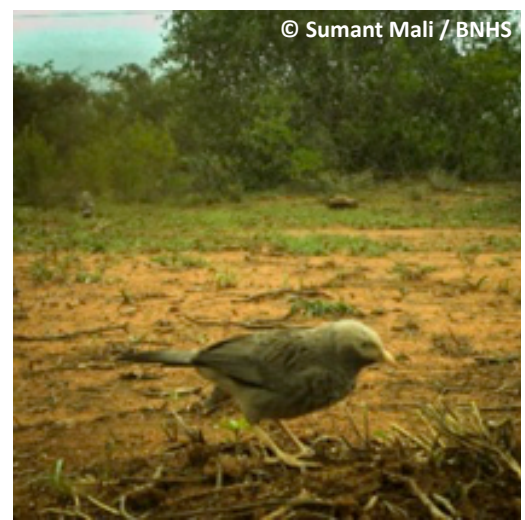

Image 52. Turdoides affinis

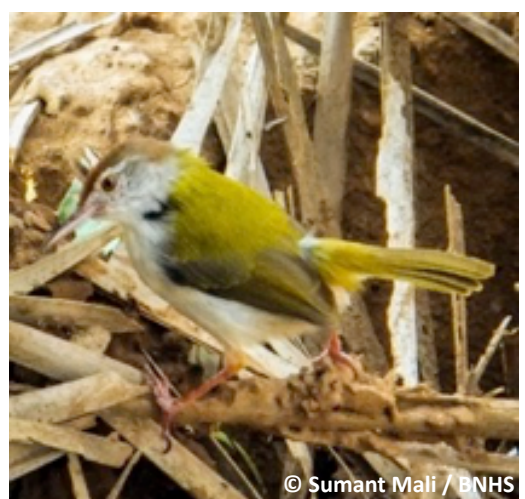

Image 55. Orthotomus sutorius

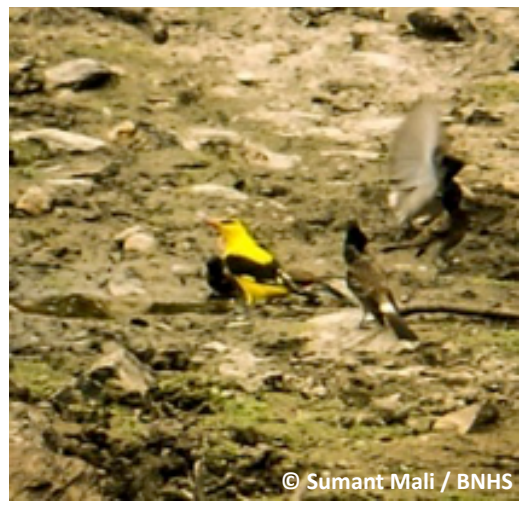

Image 58. Oriolus kundoo

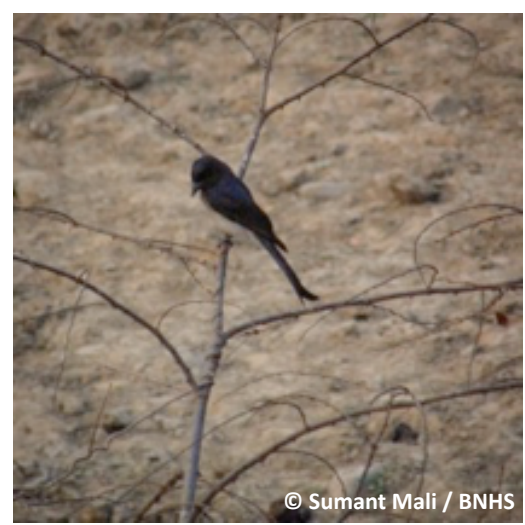

Image 61. Dicrurus caerulescens 


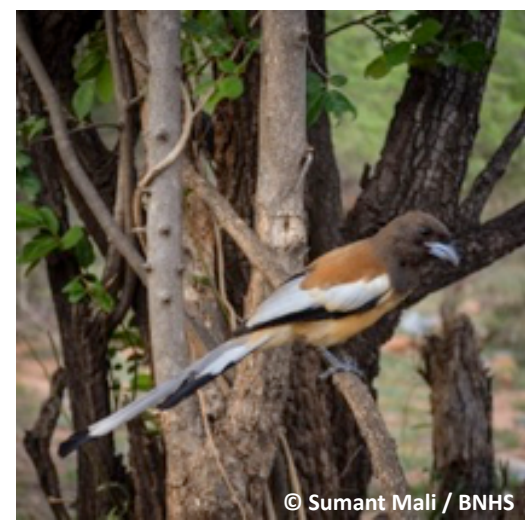

Image 62. Dendrocitta vagabunda

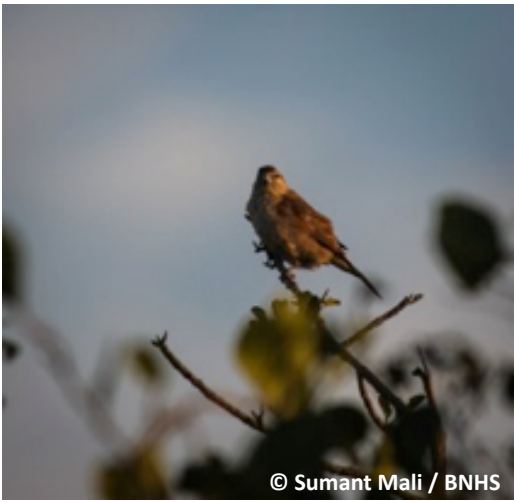

Image 65. Lonchura malabarica

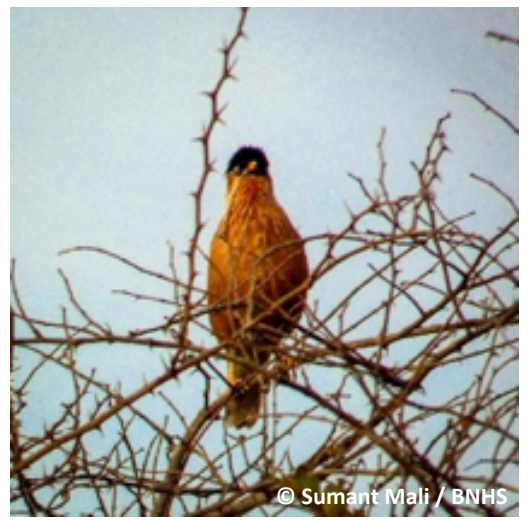

Image 63. Sturnus pagodarum

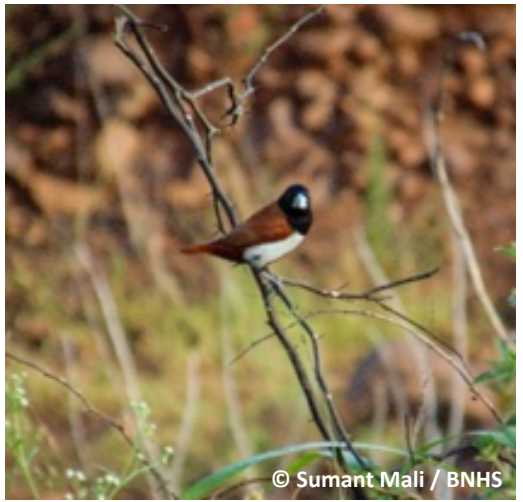

Image 66. Lonchura malacca

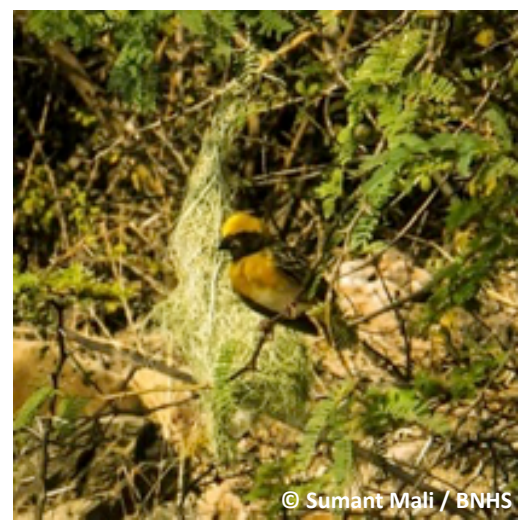

Image 64. Ploceus philippinus

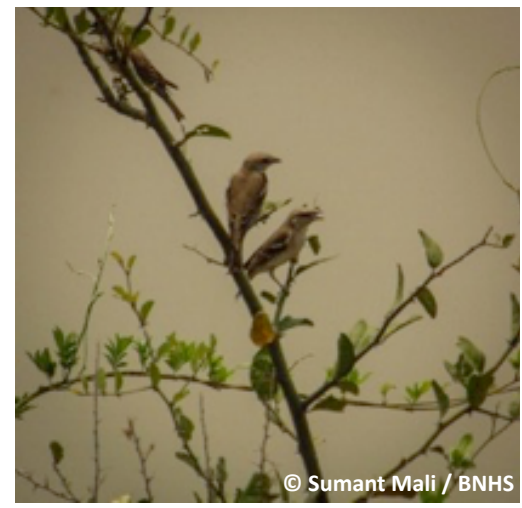

Image 67. Petronia xanthocollis

Weso 